and their QI/Program Evaluation Self-Certification Tool (https://uwmadison.col. qualtrics.com/SE/?SID = SV_3IVeNuKe8FhKc73) used as example and inspiration for this project. DISCUSSION/SIGNIFICANCE OF IMPACT: This registry was created to help understand the impact of improved management of quality projects in a hospital system. The ultimate result will be to reduce time to approve quality improvement projects, increase collaboration across the UF Health Hospital system, reduce redundancy of quality improvement projects and translate more projects into publications.

Governance for a decentralized informatics academic environment

Thomas Fogg, Margaret Demment, Jack Chang, Kathleen Holt, Dongmei Li, Helene McMurray, David Pinto and Timothy De Ver Dye University of Rochester Medical Center, Rochester, NY, USA

OBJECTIVES/SPECIFIC AIMS: Due to scope and breadth of research activity and infrastructure capacities at academic medical centers, the discipline of Biomedical Informatics is often deployed in a decentralized manner through geographically dispersed and unrelated organizational units. As a result, without a conscious strategy, an academic medical center risks redundant effort and gaps in resources, and perhaps poor coordination. A mechanism to bring together disparate organizational entities to identify, discuss, and negotiate Informatics-related concerns may produce a better institutional research environment. The University of Rochester (UR) has implemented such a strategy of Informatics governance, adapting tactics from team science, diplomacy, and deliberative engagement. METHODS/STUDY POPULATION: Based on current needs and institutional Informatics priorities, the UR's Clinical and Translational Science Institute (CTSI) established 6 Informatics "clusters" in distinct but deliberately overlapping focal areas: (I) Data - capture, management, and analysis of all types of data for research. (2) Analytics-quantitative research across the spectrum of translational research. (3) Infrastructure - technical and computing infrastructure to support informatics. (4) Electronic health records (EHR) - (i) features within the EHR explicitly designed to address the needs of research; (ii) accessing and procuring EHR data for research. (5) Population health-Informatics design and systems expertise relevant to population health research (a key CTSI focus area). (6) Education-development, deployment, and assessment of Informatics learning opportunities for learners at all levels. Each cluster facilitates access to expertise and resources around the institution, promotes collaboration, identifies redundancy, and serves as a forum to strategize regarding institutional needs related to Biomedical Informatics. A CTSI faculty or staff member leads each cluster. To maximize effectiveness of the cluster, other members are decision-makers in the organizations they represent, or serve in a critical staff function. Clusters meet in person on a quarterly basis with more frequent electronic interaction. The clusters share documents via Box, a secure online file sharing app. The cluster coordinators meet as a group on a biweekly basis to monitor progress and make plans. RESULTS/ANTICIPATED RESULTS: There were 45 different people representing 46 distinct centers, departments or offices, and 2 outside agencies agreed to participate in the clusters. In total, 20 people represented a single organizational unit; 15 represented 2 units; 8 represented 3 units, and 2 represented 4 units. The richness and complexity of these organizational linkages illustrates the decentralized nature of Informatics at the institution and the promise of the cluster approach. DISCUSSION/SIGNIFICANCE OF IMPACT: Adapting to a decentralized Informatics environment, the CTSI established clusters that recognize and respect autonomy and capacity of a wide range of units throughout the university, creating a collaborative atmosphere for steering and implementing an overall Informatics vision. As Informatics capacity rapidly expands throughout growing biomedical research institutions without a centralized Informatics hub, this distributed, deliberative approach could offer an effective governance solution that promotes cooperation. In this model, the CTSI provides the leadership and staffing necessary to ensure progress at the institutional level around Informatics and creates a venue for communication and coordination on Informatics-related topics.

Citation network towards faculty development inside and outside of CTSAs

Solomon Abiola and Kristen Bush

University of Rochester Medical Center, Rochester, NY, USA

OBJECTIVES/SPECIFIC AIMS: (I) Obtain publically available citation data, funding data, and generate multiple networks topologies based on dynamic queries of individual faculty. (2) Determine successful pathways that lead to tenure, and career advancement, in addition to determining the effect of CTSA programs on faculty collaboration. (3) Develop publically available commercial interface for the study of faculty networks METHODS/STUDY POPULATION: For our study we included all available citation and funding data publically available on all CTSA programs (as of 2015) with historical data dating back to 2005. We then included the top 25 collegiate institutions who may not have had a CTSA program (eg, Princeton University). We then developed network topologies for each university network, and explore the evolution of individuals in these networks, and the effects of faculty development-as an example in the University of Rochester network, we singled out the directors of the CTSA program there to understand their level of centrality and overall impact on network development, with key observations being that early publications across varying domains lead to stronger network performance. Although individuals who did not benefit from such development, may have succeeded but if they did were likely to leave the institution for elsewhere. RESULTS/ANTICIPATED RESULTS: A secondary goal of this project is to evaluate the effectiveness of the Clinical \& Translational Science Institute (CTSI) since its inception in 2006. The mission of CTSI is to advance the field of translational science and research, to link other departments at URMC and community stakeholders by research collaboration, publication, and goals to improve population health, and provide translational education and training to students, researchers, and physicians. To determine how the induction of CTSI affects collaboration within the URMC network, we examined the role of funding in the CTSI network. This was done around the second successful funding around 2013. In doing so we can see that not only did the funding request affect the network topology, but opened new collaborations which were not present prior to the request. DISCUSSION/SIGNIFICANCE OF IMPACT: We have developed an automated method, which is superior to manual methods necessary for citation generation and funding data analysis of faculty growth in citation networks. This technique is applicable to all institutions, not just those in a CTSA environment, but demonstrates the benefit of cross-collaborative efforts, in the case of the URMC network we can state the following. The key takeaway is for individuals to succeed in the URMC collaborative environment they should create their own network and expand it and eventually rise to prominence. There are 2 pathways to this you can take the Dewhurst approach which is to seek out collaborations among internal peers and scale up. Or you can take the Nedergaard approach which is develop the special network, and gain enough public recognition outside of the network that you are capable of leaving it (Fig. 2d). In either case, collaborations among communities and diverse out-degree networks allow faculty to succeed in their given field. Given the wealth of data which has been curated in this fashion, there are numerous explicit questions that can be asked of the data. One of the unique approaches of this data is that is highly reproducible, which allows various questions to be asked. Future work would try to determine what optimal pathways are in a given network to success, and who are ideal collaborators, and collaborations to avoid. Given this information, custom pathways to career success for individual faculty can be developed, moving beyond purely institutional level co-citation networks, which do little to advance faculty development at scale. In Figs Ic and $d$, the network increased by $75 \%$ in terms of graph density $(0.007)$ and decreased by $18.8 \%(16)$ in terms of diameter. What this suggest in that the interconnectivity of the network grew dramatically, while the ability for new members to integrate into it increased. This also apparent when one examines the modularity of the network down by $3.6 \%(0.857)$, this suggest that the network has as many communities but these communities are less isolated that those in the previous funding year, meaning fields are becoming more transdisciplinary in their collaborations. This was the result of the presence of a CTSA program, thus demonstrating the effectiveness of such institutions, however, our analysis also lays the framework for applying this to other institutions which may be considering a CTSA. Or maintaining the success of a given CTSA program, and ultimately determining where faculty should place their efforts and choose which programs to pursue career advancement.

Integration of HMIS and UI Health Cerner Clinical System to enable data sharing about homeless individuals

Neil Bahroos, Subhash Kumar Kolar Rajanna, Stephen B. Brown, Padma Thangaraj, David Melnick and Angela Freeman

Center for Clinical and Translational Science, University of Illinois at Chicago, Chicago, IL, USA

OBJECTIVES/SPECIFIC AIMS: This research project envisions the integration of Homeless Management Information System (HMIS) and UI Health Cerner electronic medical record (EMR) system with the following goals: (I) enable sharing of data about the status of the housing insecure and homeless. (2) Identify and match patient record accurately. (3) Record housing insecurity or homelessness information with structured data elements in the EMR. METHODS/STUDY POPULATION: We created a Master Person Index (MPI) of 
the homeless individuals from HMSI using OpenEMPI software package, which is an open source implementation of an Enterprise Master Patient Index (EMPI). An entity model was generated based on the selective data elements from HMIS database, which were relevant for the patient identity management and healthcare service management. An automated script was implemented to extract data from HMIS and load it into OpenEMPI to build the MPI. Once the MPI is setup, the Emergency Department users were able to perform patient identity matching and confirm housing insecure or homeless status of their patients by querying the index using the web-based tool. We developed structured data elements to record homelessness information, which will allow us to measure the prevalence of this risk among patients. We are also exploring the possibility to integrate the systems the using the IHE PIX/PDQ profile, which provides ways for healthcare applications to query a patient information server for a patient based on user-defined search criteria, and retrieve a patient's information directly into the application. RESULTS/ ANTICIPATED RESULTS: We implemented a MPI of homeless individuals, which would allow the emergency department users to perform patient identity matching of housing insecure or homeless patients, without undue privacy intrusions. We are confident that IHE PIX/PDQ profile is able to support the integration of healthcare and housing and homeless services systems and enable the data sharing in an efficient way. DISCUSSION/SIGNIFICANCE OF IMPACT: The project addressed the gap in the sharing of data about housing insecure or homeless persons between healthcare and housing and social services that will result in improvements in coordination of care, reduce the cycle time from recognition of risk to the referral to housing and services and improve health outcomes and residential stability. Successful completion of this integration project will give us a model that we can scale to many other communities.

\section{CLINICAL EPIDEMIOLOGY}

Racial differences in leukemia prognosis: New 2027 epidemiologic analysis

Shuangge Ma, Yinjun Zhao and Yu Wang

Yale School of Medicine, New Haven, CT, USA

OBJECTIVES/SPECIFIC AIMS: Research on cancer difference is of significant scientific and practical value. For leukemia, the survival disadvantage of the Blacks has been suggested in multiple studies. However, the existing epidemiologic analysis has multiple technical limitations. The goal of this study is to more accurately quantify so as to better understand different sources of racial differences in leukemia survival. METHODS/STUDY POPULATION: A new statistical method, which is based on robust regression and resampling, is developed. Data are obtained from the SEER (Surveillance, Epidemiology, and End Results) database. Using the "classic" epidemiologic methods as well as the new method, analysis is conducted on the prognosis of 4 leukemia subtypes (ALL, CLL, AML, and CML) for 4 major racial groups (White, non-Hispanic White, Black, and Asian and Pacific Islander). RESULTS/ANTICIPATED RESULTS: After effectively removing differences caused by the observed clinicopathological and demographic factors, the survival disadvantage of the Blacks persists for the following patient groups: ALL and age $>14, C L L$ and age $>14$, and $A L L$ and age $\leq 14$. The quantitative results are significantly different from those from classic epidemiologic analysis. Such observed racial differences are more attributable to the unobserved risk factors and cancer disparity. DISCUSSION/SIGNIFICANCE OF IMPACT: This study provides a more effective and more direct quantification of racial difference in leukemia prognosis. The survival disadvantage of the Blacks which is observed for certain subtypes/age groups deserves further attention but should not be overstated. More data collection and analysis are needed to more accurately decipher racial differences in leukemia and other cancer types.

2039

Modifiable risk factors Versus age on developing high predicted cardiovascular disease risk in African

Americans

Adam Bress, Lisandro D. Colantonio, John N. Booth, Tanya M. Spruill, Joseph Ravenell, Mark Butler, Amanda J. Shallcross, Samantha R. Seals, Kristi Reynolds, Gbenga Ogedegbe, Daichi Shimbo and Paul Muntner Department of Population Health Sciences, University of Utah, Salt Lake City, UT, USA

OBJECTIVES/SPECIFIC AIMS: Clinical guidelines recommend using predicted atherosclerotic cardiovascular disease (ASCVD) risk to inform treatment decisions. The objective was to compare the contribution of changes in modifiable risk factors Versus aging to the development of high 10-year predicted ASCVD risk. METHODS/STUDY POPULATION: Prospective follow-up of the Jackson Heart Study, an exclusively African-American cohort, at visit I (2000-2004) and visit 3 (2009-2012). Analyses included II I 5 AfricanAmerican participants without a high 10-year predicted ASCVD risk ( $<7.5 \%)$, hypertension, diabetes, or ASCVD at visit I. We used the Pooled Cohort equations to calculate the incidence of high ( $\geq 7.5 \%$ ) 10 -year predicted ASCVD risk at visit 3. We recalculated the percentage with a high 10-year predicted ASCVD risk at visit 3 assuming each risk factor [age, systolic blood pressure (SBP), antihypertensive medication use, diabetes, smoking, total and highdensity lipoprotein cholesterol], one at a time, did not change from visit I. RESULTS/ANTICIPATED RESULTS: The mean age at visit I was $45.2 \pm 9.5$ years. Overall, $30.9 \%$ (95\% Cl 28.3\%-33.4\%) of participants developed high 10 year predicted ASCVD risk. Aging accounted for 59.7\% (95\% Cl 54.2\%-65. $1 \%$ ) of the development of high 10-year predicted ASCVD risk compared with $32.8 \%(95 \% \mathrm{Cl} 27.0 \%-38.2 \%)$ for increases in SBP or antihypertensive medication initiation and $12.8 \%(95 \% \mathrm{Cl} 9.6 \%-16.5 \%)$ for incident diabetes. Among participants $<50$ years, the contribution of increases in SBP or antihypertensive medication initiation was similar to aging. DISCUSSION/ SIGNIFICANCE OF IMPACT: Increases in SBP and antihypertensive medication initiation are major contributors to the development of high 10 -year predicted ASCVD risk in African Americans, particularly among younger adults.

2041

Before hospice: Symptom burden, dementia, and social participation in the final years

Halima Amjad, David Roth, Jennifer Wolff, Esther Oh and Quincy Samus

Johns Hopkins University, Baltimore, MD, USA

OBJECTIVES/SPECIFIC AIMS: Traditional hospice focuses on symptoms and quality of life (QOL) at the very end of life. Clinical symptoms and $\mathrm{QOL}$ in the last I-2 years of life are also important and may be affected by dementia. Our objective was to characterize how symptoms differ between people with and without dementia in the last years before death and whether symptoms impact social dimensions of QOL. METHODS/STUDY POPULATION: We studied I 270 community-dwelling participants who died between 201 I and 2015 in the National Health and Aging Trends Study, a nationally representative cohort of older adults. From the last interview before death, we examined sensory (vision; hearing), physical (pain; problems with breathing, chewing/swallowing, speaking, upper or lower extremity strength/movement, and balance/coordination), and psychiatric (depression; anxiety; insomnia) symptoms by dementia status. We examined associations between symptoms and participation restrictions (visiting family/friends, attending religious services, participating in clubs/activities, going out for enjoyment, and engaging in favorite activity). RESULTS/ANTICIPATED RESULTS: Low energy (69\%), pain (59\%), and lower extremity strength/movement problems $(56 \%)$ were most common. People with dementia (37.3\% of decedents) had higher prevalence of all symptoms $(p \leq 0.01)$, except pain, breathing problems, and insomnia. Dementia and greater symptom burden were independently associated with greater odds of participation restrictions $(p<0.05)$. Problems speaking were significantly associated with limitations in all activities except for attending religious services. Balance/coordination, energy, and strength/movement problems were associated with limitations in 3 activities. DISCUSSION/SIGNIFICANCE OF IMPACT: Sensory, physical, and psychiatric symptoms are common in the year before death, with greater symptom prevalence in people with dementia. Both dementia and symptoms are associated with restrictions in participation. Older patients may benefit not only from earlier emphasis on palliative care but also programs and assistive devices that accommodate physical impairments.

2091

Evaluating the validity and utility of surrogate endpoints in clinical trials of chronic kidney disease (CKD)

Jian Ying, Andrew Redd and Tom Greene

The University of Utah School of Medicine, Salt Lake City, UT, USA

OBJECTIVES/SPECIFIC AIMS: The objective of this research is to determine under what conditions endpoints based on estimated glomerular filtration rate (eGFR) slope or on relatively small declines in eGFR provide valid and useful surrogate endpoints for pivotal clinical trials in chronic kidney disease (CKD) patients. METHODS/STUDY POPULATION: We consider 2 classes of surrogate endpoints. The first class includes endpoints defined by the average 